\title{
Clinical and pathological implications of miRNA in bladder cancer
}

\author{
This article was published in the following Dove Press journal: \\ International Journal of Nanomedicine \\ 20 January 2015 \\ Number of times this article has been viewed
}

\section{Cornelia Braicu,' Roxana Cojocneanu-Petric, ${ }^{1,2}$ Sergiu Chira,' Anamaria Truta, ${ }^{1,3}$ Alexandru Floares, ${ }^{4}$ Bogdan Petrut, ${ }^{5,6}$ Patriciu Achimas- Cadariu, ${ }^{7,8, *}$ loana Berindan- Neagoe ${ }^{1,9-11, *}$}

'Research Center for Functional Genomics, Biomedicine and Translational Medicine, "Iuliu Hatieganu" University of Medicine and Pharmacy, Cluj-Napoca, Romania; ${ }^{2}$ Faculty of Biology and Geology, BabeșBolyai University, Cluj-Napoca, Romania; ${ }^{3}$ Department of Medical Genetics, "Iuliu Hatieganu" University of Medicine and Pharmacy, Cluj-Napoca, Romania; ${ }^{4}$ Solutions of Artificial Intelligence Applications, Cluj-Napoca, Romania; ${ }^{5}$ Department of Urology, The Oncology Institute " Prof Dr. Ion Chiricuta”, Cluj-Napoca, Romania; 'Department of Urology, "luliu Hatieganu" University of Medicine and Pharmacy, Cluj-Napoca, Romania; ${ }^{7}$ Department of Surgery, The Oncology Institute "Prof Dr. Ion Chiricuta”, Cluj-Napoca, Romania; ${ }^{8}$ Department of Surgical Oncology and Gynaecological Oncology, "luliu Hatieganu” University of Medicine and Pharmacy, Cluj-Napoca, Romania; 'Department of Immunology, "Iuliu Hatieganu" University of Medicine and Pharmacy, Cluj-Napoca, Romania; ${ }^{10}$ Department of Functional Genomics and Experimental Pathology, The Oncology Institute "Prof Dr. Ion Chiricuta”, Cluj-Napoca, Romania; "Department of Experimental Therapeutics M.D. Anderson Cancer Center Houston, TX, USA

Correspondence: loana Berindan-Neagoe Research Center for Functional Genomics and Translational Medicine, "luliu Hatieganu" University of Medicine and Pharmacy, ClujNapoca, 23 Marinescu Street, 400I5, Romani Tel +40 264450749

Fax +40 264598885

Email ioana.neagoe@umfcluj.ro

Patriciu Achimas-Cadariu

Department of Surgery, The Oncology Institute "Prof Dr. Ion Chiricuta", Cluj-Napoca, Romania Tel +40 264450749

Fax +40264598885

Email pachimas@umfcluj.ro
Abstract: MicroRNAs (miRNAs) are small, noncoding RNA species with a length of 20-22 nucleotides that are recognized as essential regulators of relevant molecular mechanisms, including carcinogenesis. Current investigations show that miRNAs are detectable not only in different tissue types but also in a wide range of biological fluids, either free or trapped in circulating microvesicles. miRNAs were proven to be involved in cell communication, both in pathological and physiological processes. Evaluation of the global expression patterns of miRNAs provides key opportunities with important practical applications, taking into account that they modulate essential biological processes such as epithelial to mesenchymal transition, which is a mechanism relevant in bladder cancer. miRNAs collected from biological specimens can furnish valuable evidence with regard to bladder cancer oncogenesis, as they also have been linked to clinical outcomes in urothelial carcinoma. Therefore, a single miRNA or a signature of multiple miRNAs may improve risk stratification of patients and may supplement the histological diagnosis of urological tumors, particularly for bladder cancer.

Keywords: bladder cancer, miRNA, prognostic, diagnostic

\section{Introduction}

Bladder cancer most commonly refers to carcinoma of the epithelial lining of the urinary bladder, the urothelium. Its symptoms are nonspecific and include hematuria, discomfort during urination, and higher frequency and urgency of urination. Because any or all of these symptoms can be caused by a number of other medical conditions, including fairly common urinary tract infections, a clear diagnosis may be achieved only by microscopically examining the cells either from urine or from tissue collected from the bladder or by cystoscopy. This is an invasive procedure that sometimes requires the administration of an anesthetic, especially when a biopsy is necessary. The noninvasive diagnosis methods, which consist of examining the cells that were shed into the urine, is not always reliable, as the quantity of cells depends on the stage of the disease, with many fewer cells observed in the earlier phases. ${ }^{1}$

Although bladder cancer incidence has not increased in recent years in most Western developing countries, its rates are still high. According to Globocan 2012, bladder cancer is the sixth most prevalent type of cancer in men, accounting for $4.4 \%$ of cases. The incidence in women is about threefold lower, but for both sexes, it ranks ninth in cancer incidence worldwide (3\%). Regarding mortality rates, it is the ninth leading cause of death by cancer among men and the 13 th for both sexes. ${ }^{2}$

In the United States, an estimated 54,610 new cases of bladder cancer were expected to occur in men in 2013, and 17,960 women presumably acquired this malignancy. The probability of developing this disease seems to increase with age, growing from a $0.02 \%$ chance of having bladder cancer by the age of 39 years to a $3.69 \%$ ( 1 in 27$)$ 
chance of having this malignancy over the age of 70 years. ${ }^{3}$ According to the same source, the 5 year relative survival rates in relation to the stage at the time of diagnosis are very high for in situ bladder carcinomas (96\%) but go down to around $6 \%$ for advanced stages with distant metastases (as seen in Figure 1). Regarding the mortality caused by this illness, a total number of 15,210 fatalities were expected to be attributed to bladder cancer in the United States during 2013 , of which $71 \%$ are men. ${ }^{4}$

It was observed that $75 \%$ of diagnosed bladder cancer cases are localized in the mucosa or submucosa (non-muscleinvasive bladder cancer), whereas in $25 \%$ of situations, the tumor cells have already infiltrated the muscle (muscleinvasive bladder cancer [MIBC] $).^{5}$

This malignancy has a complex and multifactorial etiology, incorporating both genetic and environmental causes. Two main types of bladder cancers can be described: lowgrade superficial tumors (papillary and multifocal, which occasionally progress to invasive disease and have a good prognosis) and high-grade invasive cancer (nodular, metastasizes in the early phases, and has a poor prognosis).

According to its stage, bladder cancer presents itself in a different manner. In staging bladder cancer, the most widely used approach is the TNM system, where T stands for tumor and indicates the size and the degree of tumor penetration in adjacent tissue, $\mathrm{N}$ displays the amount of invasion to nearby lymph nodes, and $\mathrm{M}$ shows whether the cancer has metastasized to distant areas of the body. Each of the letters is assigned numbers that describe how invasive the malignant cells are and give more details about the precise localization of the cancer. Thus, cancer that is confined to the urothelium is considered to be Stage Tis, or carcinoma in situ, if the cancer is high grade, or Stage Ta, or noninvasive papillary tumor, if the cancer is low grade. Grading refers to the degree of aggressiveness of the cancer cells, with low-grade cancer

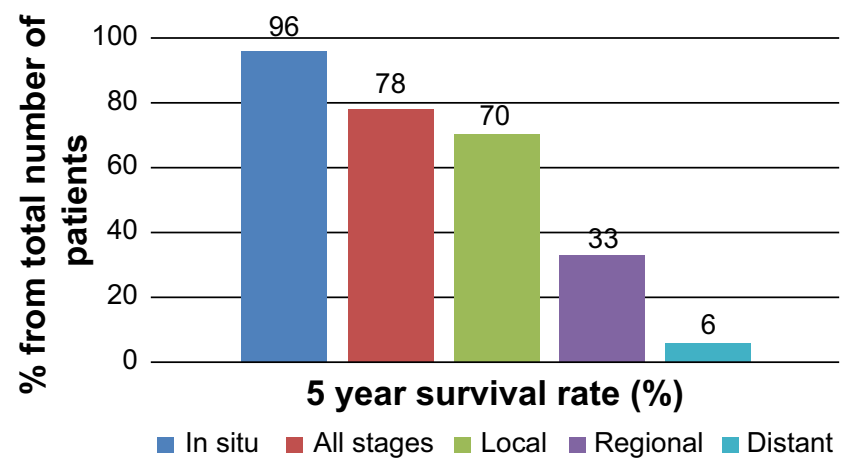

Figure I Five-year relative survival rates for bladder cancer in relation to the stage at time of diagnosis. being the less aggressive cells, which grow slower, look quite normal, and act similar to healthy cells, and with high-grade cancer being characterized by fast-growing cells, which look and act in a disorganized manner and are more likely to progress into the muscle layer of the bladder. T-stages 1 through 4 represent the degree to which the cancer has grown in the adjacent layers of tissue, from the connective tissue just beneath the urothelium (T1) to tissue structures located outside the bladder (T4). Regarding the potential spread of cancer cells to the lymphatic system, or to the lymph nodes to be more precise, the N-stages of bladder cancer describe this phenomenon with the help of four stages. Thus, this type of staging varies from N0, with no cancer found in any lymph node, to N3, where the cancer has spread to one or more of the lymph nodes located in the groin area. In some cases, bladder cancer spreads to nodes found in the abdomen or even further up in the body. The M-stages of bladder cancer describe whether the cancer has metastasized to another organ in the body (M1) or has not (M0). When it metastasizes, bladder cancer most likely spreads to the lungs, liver, or bones.

\section{Clinical and pathological implication of micro-RNA in bladder cancer}

The challenges that clinicians face when caring for patients with bladder cancer are the difficulties of early diagnosis, disease recurrence, and progression. Current prognostic strategies, such as tumor grade, stage, size, and number of foci, have restricted utility for clinicians because they do not specifically exhibit the clinical outcomes. ${ }^{6}$ Diagnosis and monitoring strategies for bladder cancer have been based on the integration of cystoscopy and urinary cytology data. ${ }^{7}$ As a consequence, researchers have been searching for novel biomarkers, and an important research direction was focusing on the role of microRNAs (miRNAs) in the development of bladder cancer (Figure 2).

\section{miRNA definition}

miRNAs, small RNA molecules that are not translated into proteins, are 18-22 nucleotide long transcripts that were proven to have active roles in negatively regulating the expression of genes resulting from their partial complementarity to the $3^{\prime}$ untranslated region of target messenger RNAs. These miRNAs are not only differentially expressed in malignant versus normal tissue but also vary between different types of cancer. ${ }^{8,9}$ Studies have shown that miRNAs are active players in various cell activities, among which are apoptosis, cell development, proliferation, and differentiation (Figure 3). Because of their implication in different processes 


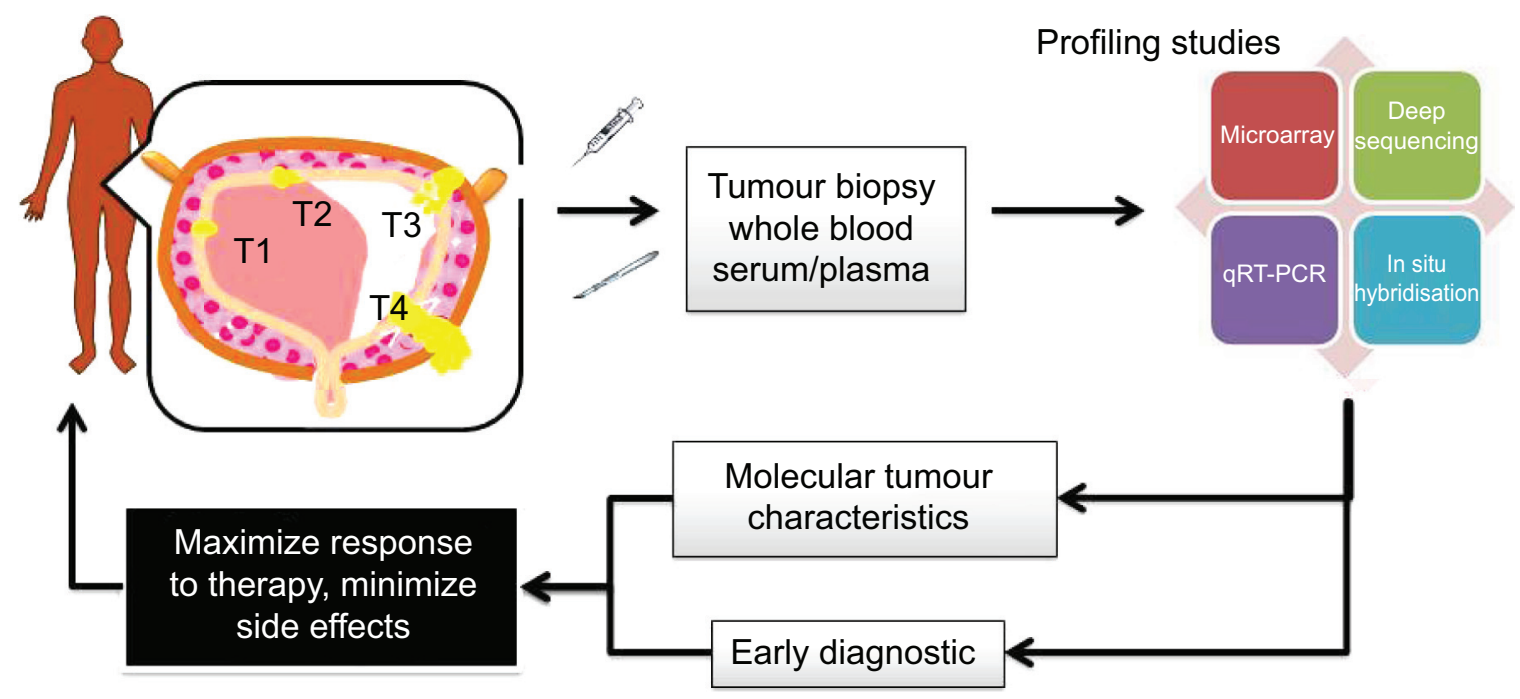

Figure 2 Role of micro-RNA signature in bladder cancer diagnostic and prognostic.

Abbreviation: $q R T-P C R$, quantitative reverse transcription polymerase chain reaction.

at the cellular level, miRNAs have been attributed the potential of becoming biological markers for early diagnosis or prognostication, as well as the potential for using them as therapeutic targets for different oncological diseases. ${ }^{7,10,11}$

One of the reasons for the biomarker potential of miRNAs is the fact that their profiles were shown to be altered in tumor cells compared with normal tissue at different levels, including miRNA regulation of transcription, miRNA maturation processes, and alteration at genetic and epigenetic levels.
miRNA dysregulation leads to the idea that these molecules have the potential to act as oncogenes, as well as tumor suppressors. Just as in the case of oncogenes, when oncogenic miRNAs are upregulated in cancer, they lead to the downregulation of tumor suppressor genes or to the inhibition of genes with roles in cell cycle, cell proliferation, or apoptosis. Following the same principle, tumor-suppressive miRNAs contribute to the downregulation of oncogenes. Understanding these mechanisms has great importance

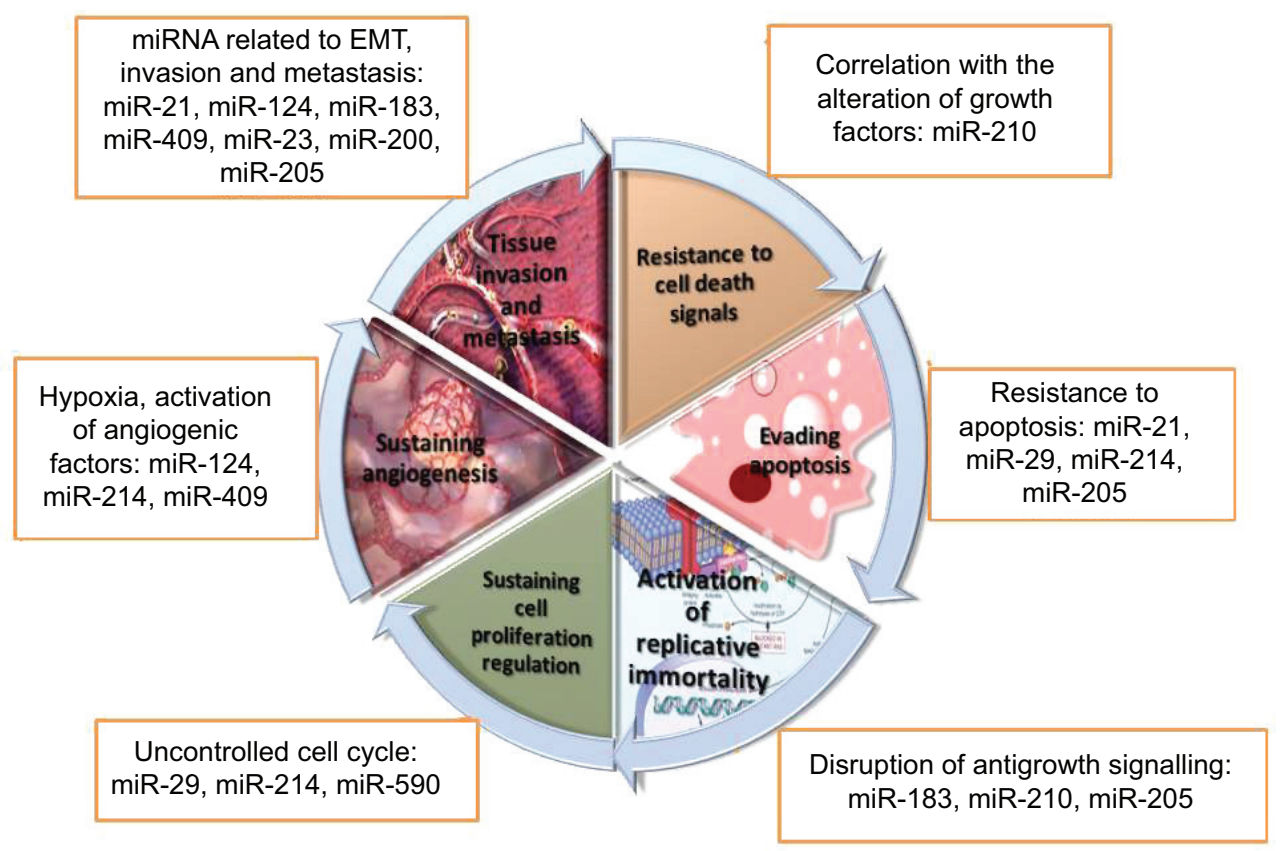

Figure 3 Hallmarks of bladder cancer beyond miRNA perspective. Abbreviations: miRNA, micro-RNA; EMT, epithelial-mesenchymal transition. 
in bringing the scientific community one step closer to deciphering how miRNAs are involved in tumorigenesis, ${ }^{8}$ which can consequently contribute to the discovery of ways to tamper with angiogenesis, invasion and metastasis, and the immune response.

Expression profiles of miRNAs follow the same alteration patterns in different types of cancers, which suggests they are involved in signaling pathways that are modified in cancer. Some of these correlations between altered miRNA expression patterns and cancer pathogenesis have been described in the literature, but further studies are necessary to unravel information on the degree of involvement of altered miRNA levels on intracellular targets. ${ }^{7-9}$

To counteract the effects of oncogenic miRNAs and to decrease their cellular involvement, the most suitable approach is the development of various therapeutic strategies, whereas in the case of tumor-suppressive miRNAs, the tactic to restore biologic activity would involve bringing target mRNA levels to their original state..$^{5-9}$

\section{Bladder cancer miRNA profiling studies to discriminate malignant from normal tissue}

The miRNA alterations profile is connected with the alteration of the normal phenotype and is able to predict tumor progression. To date, the role of miRNA mechanisms of bladder cancer carcinogenesis has not been completely deciphered, but as in the case of many other cancers, it is characterized by an alteration of the molecular profile caused by the accumulation of genetic and epigenetic changes. $^{12}$

Evaluation of global expression patterns of miRNAs provides key opportunities with important practical application, taking into account the fact that they modulate key biologic processes. ${ }^{13}$ The expression pattern of miRNAs varies between tissues and developmental stages, and several miRNAs are related to the disease stage, not only in a tissue-specific manner. The evaluation of miRNA patterns will lead to a better diagnostic strategy or to the development of methods to assess prognosis and the response to treatment. As in other cancer types, a distinct miRNA signature among normal tissue was observed, even in the case of carcinoma in situ or among the different stages of the disease.

There are several studies focused on the identification of miRNAs involved in bladder carcinogenesis with prognostic or diagnostic potential or with therapeutic implications (Table 1). Another study performed on 166 non-muscleinvasive bladder cancer cases and $80 \mathrm{MIBC}$ cases led to the identification of the particular signature of 15 miRNAs that can differentiate normal from malignant phenotypes. ${ }^{14}$ The same study observed the upregulation of miR-146b and miR-9 only in the case of MIBC.

A recent deep sequencing evaluation of nine bladder cancer samples revealed 656 differentially expressed miRNAs; respectively, antisense sequences ( $\mathrm{miRNA} * \mathrm{~s}) .{ }^{15} \mathrm{Hsa}-\mathrm{miR}-96$ displayed the highest statistically significant overexpression value, and miR-490-5p presented the highest downregulation value.

Evaluation of miRNA signatures provided the means to distinguish among muscle-invasive bladder cancer, nonmuscle-invasive bladder cancer, and noncancerous controls, using microarray technology in a small study on only 20 bladder cancer and 18 control patients. ${ }^{16}$ Despite the limitations caused by the low number of participants, this proofof-concept study provides preliminary data that suggest the practicality of this method for the early detection of bladder cancer. A screening study performed by Ratert et al, ${ }^{5}$ using microarray technology followed by a validation step using quantitative reverse transcription polymerase chain reaction (qRT-PCR), reveals a specific miRNA signature containing 15 miRNAs, of which seven (miR-100, miR-125b, miR130a, miR-139-5p, miR-145*, miR-199a-3p, miR-214, and miR-222) were upregulated and eight were downregulated ${ }^{5}$ in tumor tissue versus healthy tissue. Furthermore, miR20a, miR-106b, miR-141, and miR-205 were found to be upregulated in bladder cancer, ${ }^{17,18}$ in contrast to an inverse expression behavior in other locations such as renal or prostate cancer. ${ }^{18,19}$

\section{miRNA profiling studies in bladder cancer patient stratification}

The critical roles of miRNAs in cancers are gradually explored, and their applications as potential therapeutic targets have generated great interest in developing novel strategies for treating cancer. miRNA alteration profile is connected with the alteration of the normal phenotype, and thus it may be involved in the prediction of tumor progression. ${ }^{20,21}$ To date, the role of miRNA mechanisms in bladder cancer carcinogenesis has not been completely deciphered. Still, similar to other malignancies, bladder cancer is characterized by an alteration of the molecular profile caused by the accumulation of genetic and epigenetic modifications. ${ }^{22,23}$ This has raised the interest of several research groups in using miRNA in diagnostic and prognostic ways, and recently, a greater amount data on this subject became available. In one of these studies, it was observed that the inhibition of the miR-200 family, miR-205, 


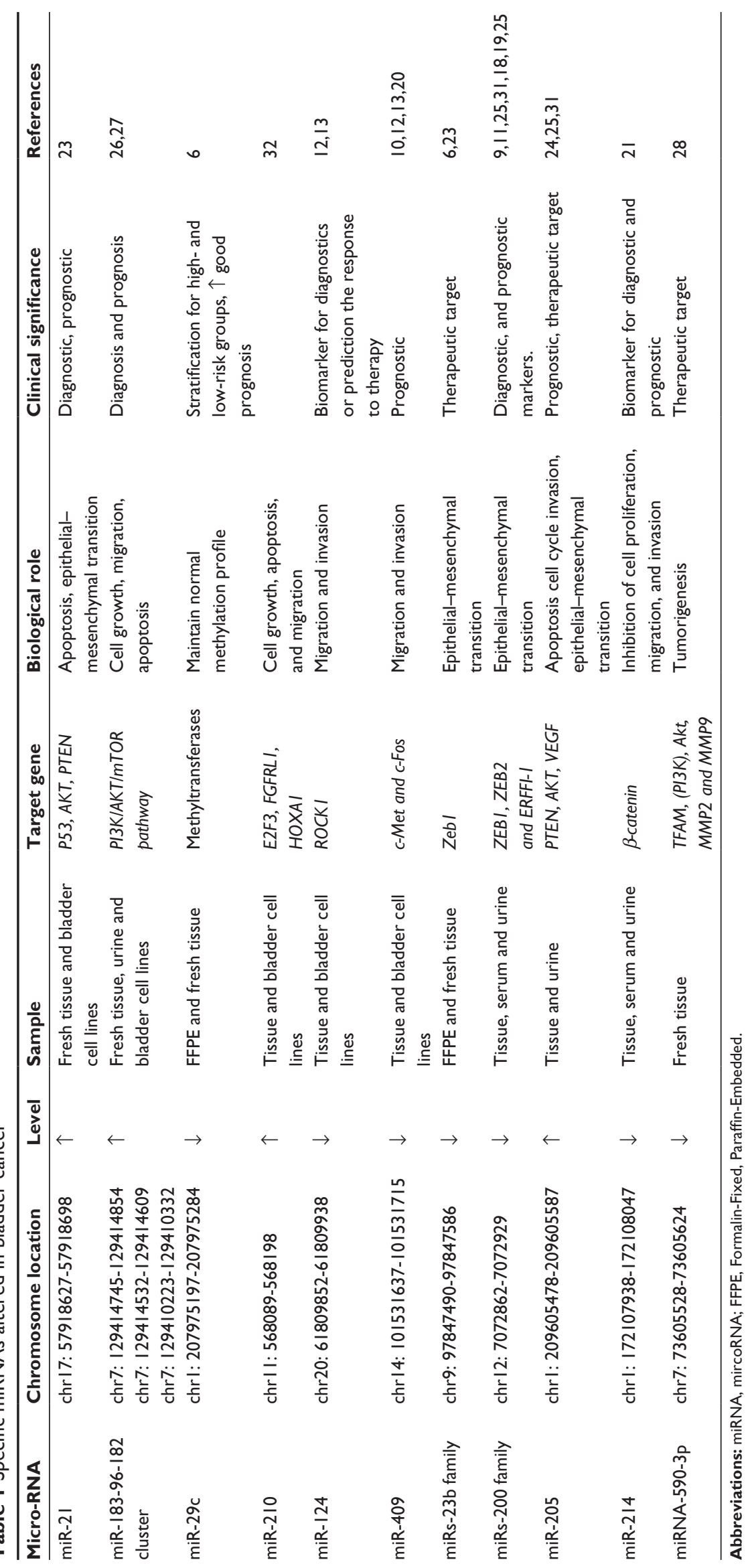


miR-152, and miR-10a, was connected to an increased DNA methylation pattern in normal versus tumor tissue. ${ }^{24-26}$ At the same time, miR-200c downregulation is related to disease progression and poor outcome. ${ }^{20} \mathrm{miR}-9$, miR-182, and miR$200 \mathrm{~b}$ were identified as being related to tumor aggressiveness and prognosis in bladder cancer. ${ }^{27}$ miR-100 downregulation presents unfavorable prognosis, and therefore can be used as biomarker for risk stratification. ${ }^{28}$

miR-205 was observed as being able to discriminate between low-grade papillary urothelial carcinoma and highgrade papillary urothelial carcinoma, whereas miR-145 distinguished high-grade papillary urothelial carcinoma from infiltrating carcinoma. ${ }^{29}$ In another study, HG was connected with miRNA-21 upregulation that suppresses p53 function, and low-grade urothelial carcinoma has been associated with the downregulation of miRNAs-99a/100; at the same time, miR129, miR-133b, and miR-518c* were identified as potential markers for disease progression, and miR-452 and miR-452* were shown to be markers with prognostic value. ${ }^{30}$

In a study conducted by Wang et $\mathrm{al}^{9}$ on a group of patients with confirmed bladder cancer, the expression levels of miR$200 \mathrm{c}$ and miR-141 were altered in the urine sediment of these patients, with the values being normalized after surgery. ${ }^{9}$ Other particular signatures, such as miR-21 and miR-373 overexpression, were observed for high-grade, muscleinvasive bladder cancer when compared with normal tissue, targeting p53 pathway, or epithelial-mesenchymal transition (EMT). ${ }^{31}$ In contrast, overall increased expression of miRNAs is observed in high-grade, muscle-invasive bladder cancer compared with adjacent normal bladder urothelium, including miRs predicted to target $\mathrm{p} 53$, such as miR-21 and miR-373. Furthermore, p53 inhibits transcriptional factors that sustain mesenchymal differentiation, such as ZEB-1 and ZEB-2, by modulating members of the miR-200 family.

Fibroblast growth factor 3 regulates important cellular processes, such as cell growth, differentiation, and angiogenesis, which are important in bladder cancer development and progression. ${ }^{32,33}$ Some studies presented the relationship with outcome possibly because of different its related miRNA target such as miR-145, miR-101, miR-100, and miR-99a being characteristic for low-grade, non-muscle-invasive bladder cancer. ${ }^{23}$

The miR-183-96-182 cluster was shown to be upregulated in many human cancers, such as breast, lung, and prostate, but also in bladder malignant tumors. ${ }^{34}$ In a recent study using synthetically designed miRNA-mowers, researchers proved the ability of the miR-183-96-182 cluster and miR210 to reduce cell proliferation, to activate the apoptotic machinery, and to inhibit migration. ${ }^{35}$ Knockdown of the miR-183/96/182 cluster targeted the PI3K/AKT/mTOR pathway and subsequently enhanced apoptosis. miR-210 is recognized as a novel biomarker for hypoxia, but an in vitro study using anti-miR-210 suggested it was connected with reduced proliferation and with migration and an activation of apoptosis. ${ }^{27}$ Other miRNAs have been proved to act as key factors in tumorigenesis. One example is miR-590-3p, which is negatively regulated by mitochondrial transcription factor $\mathrm{A}$ (TFAM). ${ }^{36}$ TFAM has an active role in mitochondrial DNA replication and transcription, whereas miRNAs are important regulators of gene expression. However, the exact means by which TFAM and particular miRNAs associate to promote cancer development still need to be deciphered. Nonetheless, this particular study reported that TFAM expression was considerably amplified in bladder cancer, whereas the expression of miRNA-590-3p was decreased.

EMT is an essential mechanism activated during cancer invasion and metastasis and is characterized by loss of cell adhesion, inhibition of E-cadherin expression, and increased cell mobility. ${ }^{37}$ Members of the miR-200 family appear to control the EMT by targeting ZEB1, ZEB2, and EGFR. This fact has important clinical applications that may lead to the development of novel EGFR therapy by modulation of miR$200 .{ }^{29}$ At the same time, loss of miR-200 expression promotes EMT and resistance to EGFR-based therapies. ${ }^{22}$

Another important aspect was the identification of a specific miRNA signature able to predict drug resistance. miRNAs 1290, 138, let-7i, and let-7b, via mucin-4, proved to be connected with resistance to gemcitabine in in vitro models. ${ }^{38}$ In another study, it was observed that miRNA-27a is involved in cisplatin-resistant bladder cancer. ${ }^{39}$

miR-126 was downregulated in tumor tissue versus normal tissue and, moreover, was observed to target ADAM9, a key gene related to angiogenesis. ${ }^{40}$ Another study revealed that miR-1280 targets oncogene $R O C K 1$, leading to the reducing of migration and invasion, mechanisms that are related to the metastasis. ${ }^{41}$ It was observed that downregulation of miR-195 and miR-497 is related to bladder cancer progression and metastasis. ${ }^{42}$ In a bladder cancer animal model study, it was observed that miR-582 delivery was connected with the inhibition of tumor growth and metastasis. ${ }^{43}$

\section{miRNA as biomarkers for diagnostics and prognostication in bladder cancer}

According to its definition, a biomarker should be a stable molecule that is accessible, inexpressive to assess, and 
disease-specific. Lately, a lot of studies have indicated that miRNAs are a novel class of cancer biomarkers. ${ }^{44}$ The circulating miRNAs were proposed as reliable markers of the urological state. ${ }^{8,10}$ The characterization of miRNAs' altered pattern appears to be an alternative to serum proteins, and significant recent experimental data have focused on analyzing and describing miRNAs circulating in biological fluids. ${ }^{45}$ Together with serum/plasma samples, one biological fluid that has significant clinical relevance for this pathology is represented by urine.

miRNAs in biological specimens can furnish valuable information with regard to bladder cancer oncogenesis; hence, the development and implementation of miRNAbased screening methods are anticipated to gradually become a standard approach in clinical diagnostic, as an integrated part of personalized medicine. The discovery of novel methods developed in the last years allows the quantification of miRNA in low amounts, as in the case of biological fluids that are used for biomarker screening.

Bladder cancer diagnosis is based on the detection of cancer cells in a urine specimen collected from the patient. ${ }^{46} \mathrm{Con}-$ sidering that urine passes via the normal tissue or malignant lesions in the urinary tract, its miRNA content is connected with the disease status as a result of the presence of tissue with an altered phenotype. Urinary biomarkers, including miRNAs, may be related to multiple urologic pathologies (such as prostate or renal cancer), not only bladder cancer, and hence it is important to develop specific ways to accurately differentiate among them. ${ }^{32}$

Some of these miRNAs have the potential of becoming biomarkers for bladder cancer diagnosis and prognosis, as well as for predicting treatment targets. ${ }^{47}$ For instance, miR-143, miR-222, and miR-452 detected in urine specimens were clinically useful for noninvasive bladder cancer diagnostics, ${ }^{6}$ and miR-9, miR-182, and miR-200b were found to be related to bladder tumor aggressiveness and survival. ${ }^{48}$

Moreover, miR-141 and miR-205 were associated with overall survival time,${ }^{5}$ underlining the potential of miRNAs to function as diagnostic and/or prognostic markers for bladder cancer. Studies show that miR-9, miR-182, and miR$200 \mathrm{~b}$ appear to be related to MIBC tumor aggressiveness and recurrence-free and overall survival. ${ }^{5,11}$ Ratert et al ${ }^{5}$ observed a correlation between miR-141 and miR-205 expression levels with the overall survival of the patients. Other studies revealed that some miRNAs, particularly miR-126, miR-182, and miR-199a could be found in larger quantities in the urine of bladder cancer patients than in the urine of controls, either healthy subjects or patients with infections. Wang et $\mathrm{al}^{11}$ observed a decreased representation of miR200 family members (miR-141, miR-155, and miR-429) in the urine of bladder cancer patients, whereas after tumor resection, the levels of these particular miRNAs increased considerably. The same observation was made during other studies, which emphasizes the potential utility of the miR200 family in diagnostic or prognostic situations. ${ }^{33}$

The results of a study conducted by Hanke et $\mathrm{al}^{49}$ displayed a higher stability of urinary miRNAs, supporting the development of a screening method for miRNA-126 and miRNA-182 with encouraging diagnostic potential. A recent study performed by $\mathrm{Li}$ et $\mathrm{al}^{20}$ on 138 non-muscleinvasive bladder cancer patients versus 144 healthy controls revealed higher urinary levels of cell-free miR-214 in cancer than in the control groups, emphasizing the potential utility of miR-214 as independent prognostic marker for disease recurrence.

In a 2012 study, Wang et $\mathrm{al}^{11}$ assessed the expression levels of several miRNAs involved in EMT on 51 patients with bladder cancer and 24 controls. This study showed that the miR-200 family, miR-192, and miR-155 were downregulated in the urinary sediment, thus being correlated with the expression of EMT urinary markers (ZEB1, vimentin, TGF- $\beta 1$, and Ras). ${ }^{9}$

Another pilot study revealed that miR-26b-5p, miR144-5p, and miR-374-5p were upregulated in the case of invasive carcinoma when compared with the control group, and at the same time, it pinpointed the overexpression of miR-618 and miR-1255b-5p in the urine collected from the cases with aggressive tumors. ${ }^{50} \mathrm{miR}-96$ and $\mathrm{miR}-183$ expression levels in the urine of bladder cancer patients were significantly higher than in the case of healthy patients. This overexpression was correlated with tumor grade and pathological stage. ${ }^{51}$

\section{Clinical trials}

During the last decade, an ever-increasing number of studies have been conducted on the implication of miRNAs in the initiation and progression of the malignant phenotype, as well as on their roles and possible uses for early diagnosis, prognosis, and prediction of response to therapy. Among these studies, only some are focused on the research of miRNA involvement in bladder cancer. For instance, a search on the National Center for Biotechnology Information's PubMed using the string "bladder cancer" AND "miRNA" returned more than 200 articles.

However, from the clinical, practical perspective, the importance of these studies can be quantified in their translational abilities to transcend from the bench to the 
Table 2 Overview of the query results returned by different searches regarding bladder cancer on the ClinicalTrials.gov webpage

\begin{tabular}{|c|c|c|}
\hline Initial search query & $\begin{array}{l}\text { Additional } \\
\text { key words }\end{array}$ & $\begin{array}{l}\text { Number of clinical } \\
\text { trials }\end{array}$ \\
\hline "bladder" AND “cancer" & - & 674 \\
\hline "bladder" AND “cancer” & Biomarker & 93 \\
\hline "bladder” AND “cancer” & Signature & 27 \\
\hline "bladder" AND "cancer" & Diagnosis & 90 \\
\hline "bladder" AND "cancer" & Treatment & 542 \\
\hline "bladder” AND “cancer” & Therapy & 481 \\
\hline "bladder" AND “cancer” & MicroRNA/miRNA & I \\
\hline "bladder" & MicroRNA/miRNA & 2 \\
\hline
\end{tabular}

Abbreviation: miRNA, mircoRNA.

bedside, and the first step toward achieving this takes the form of clinical trials. The National Institutes of Health provides an online database (http://clinicaltrials.gov/) in which they publish, according to their own homepage, "publicly and privately supported clinical studies of human participants conducted around the world." It currently lists 155,545 clinical trials, located in all 50 states and in 185 countries around the world, and is thus one of the most valuable sources for centralized information on this topic. In our attempt to find out the share of clinical trials focused on urinary bladder malignancies, we first searched this database using the search query "bladder" AND "cancer," which returned a total number of 674 studies in the "Conditions" field. We further refined the search using different additional key terms, and the results returned by these searches are presented in Table 2.

As can be observed, the returned results for clinical trials that included references to both bladder cancer and miRNAs consisted of only one study, which is actually not focused on bladder cancer per se but, rather, on prostate adenocarcinoma. The name of the trial is "Hypofractionated, Dose Escalation Radiotherapy for High Risk Adenocarcinoma of the Prostate," and the only reference to bladder cancer is made in "Secondary Outcome Measures," where the authors mention the correlation of "dose-volume histograms of the rectum and bladder by studying wall and whole organ volumes to the development of GI (gastro-intestinal) and GU (genitourinary) toxicity." In the description of this study, miRNAs are not the main focus of this study, being mentioned only once, as well in the context of secondary measures, more precisely when discussing the predictive and prognostic value of various biological variables, which in this case is the expression of X-linked miRNAs.

To see whether there are any other studies on miRNA implication in bladder diseases in general, we relaxed the search, using only the key words "bladder" and "miRNA."
This inquiry returned two results, of which one was the trial we already mentioned, and the second has the title "The Role of microRNAs in Organ Remodeling in Lower Urinary Tract Dysfunction" and does not make any reference to malignant pathology. So it is safe to assert the fact that for the time being, there are no clinical trials focused on the implication of miRNAs in bladder cancer or on topics that are connected to our subject of research.

\section{Conclusion}

Distinct miRNAs expression signatures were observed among the different stages and histological subtypes of bladder cancer. This miRNA altered pattern may provide knowledge into the molecular pathways affected in bladder carcinogenesis being investigated by several research teams. At this time, there is an increasing amount of literature data, but the implementation into the clinic remains limited. This may be because of the higher heterogeneity of biological specimens and lack of confirmed methods for in vitro and in vivo validation of the in silico miR data. Despite that, miRNAs might be promising biomarkers for the prognostic and diagnostic features of bladder cancer.

Accumulation of novel data concerning miRNA signatures holds great potential, permitting progress leading to an accurate diagnosis. In this context, the clinician might furnish an appropriately optimized treatment scheme impairing the tumors, as well as a better quality of life.

\section{Acknowledgments}

This study was financed by the PN-II-PT-PCCA-2011-3.11221: "Intelligent Systems of Prediction of Recurrence and Progression in Superficial Bladder Cancer Based on Artificial Intelligence and Microarray Data: Tumor mRNA and Plasmatic microRNA-IntelUro.” AT received a fellowship financed by Grant 159/1.5/s/138776.

\section{Disclosure}

The authors report no conflicts of interest in this work.

\section{References}

1. Urquidi V, Rosser CJ, Goodison S. Molecular diagnostic trends in urological cancer: biomarkers for non-invasive diagnosis. Curr Med Chem. 2012;19(22):3653-3663.

2. International Agency for Research on Cancer and the World Health Organization. The GLOBOCAN Project. Available from: http://globocan.iarc. fr/. Accessed December 5, 2014.

3. National Cancer Institute. Surveillance Research Program. Bethesda, MD: National Cancer Institute. Available from: http://surveillance. cancer.gov/. Accessed December 5, 2014.

4. American Cancer Society. Cancer Facts and Figures 2013. Atlanta: American Cancer Society; 2013. 
5. Ratert N, Meyer HA, Jung M, et al. miRNA profiling identifies candidate mirnas for bladder cancer diagnosis and clinical outcome. J Mol Diagn. 2013;15(5):695-705.

6. Rosenberg E, Baniel J, Spector Y, et al. Predicting progression of bladder urothelial carcinoma using microRNA expression. BJU Int. 2013;112(7):1027-1034.

7. Majid S, Saini S, Dahiya R. Wnt signaling pathways in urological cancers: past decades and still growing. Mol Cancer. 2012;11(1):7.

8. Heneghan HM, Miller N, Kerin MJ. MiRNAs as biomarkers and therapeutic targets in cancer. Curr Opin Pharmacol. 2010;10(5): 543-550.

9. Croce CM. Causes and consequences of microRNA dysregulation in cancer. Nat Rev Genet. 2009;10(10):704-714.

10. Huang $\mathrm{S}, \mathrm{He} \mathrm{X}$. The role of microRNAs in liver cancer progression. $\mathrm{Br}$ J Cancer. 2011;104(2):235-240.

11. Wang D, Qiu C, Zhang H, Wang J, Cui Q, Yin Y. Human microRNA oncogenes and tumor suppressors show significantly different biological patterns: from functions to targets. PLoS One. 2010;5(9):e13067.

12. $\mathrm{Hu} \mathrm{Z}$, Lin $\mathrm{Y}$, Chen $\mathrm{H}$, et al. MicroRNA-101 suppresses motility of bladder cancer cells by targeting c-Met. Biochem Biophys Res Commun. 2013;435(1):82-87.

13. Xu X, Li S, Lin Y, et al. MicroRNA-124-3p inhibits cell migration and invasion in bladder cancer cells by targeting ROCK1. J Transl Med. 2013;11(1):276.

14. Pignot G, Cizeron-Clairac G, Vacher S, et al. microRNA expression profile in a large series of bladder tumors: identification of a 3-miRNA signature associated with aggressiveness of muscle-invasive bladder cancer. Int J Cancer. 2013;132(11):2479-2491.

15. Han Y, Chen J, Zhao X, et al. MicroRNA expression signatures of bladder cancer revealed by deep sequencing. PLOS ONE. 2011;6(3): e18286.

16. Adam L, Wszolek MF, Liu CG, et al. Plasma microRNA profiles for bladder cancer detection. Urol Oncol. 2013;31(8):1701-1708.

17. Xu X, Chen H, Lin Y, et al. MicroRNA-409-3p inhibits migration and invasion of bladder cancer cells via targeting c-Met. Mol Cells. 2013;36(1):62-68.

18. Schaefer A, Jung M, Mollenkopf HJ, et al. Diagnostic and prognostic implications of microRNA profiling in prostate carcinoma. Int J Cancer. 2010;126(5):1166-1176.

19. Wotschofsky Z, Liep J, Meyer HA, et al. Identification of metastamirs as metastasis-associated microRNAs in clear cell renal cell carcinomas. Int J Biol Sci. 2012;8(10):1363-1374.

20. Li S, Xu X, Xu X, et al. MicroRNA-490-5p inhibits proliferation of bladder cancer by targeting c-Fos. Biochem Biophys Res Commun. 2013;441(4):976-981.

21. Kim SM, Kang HW, Kim WT, et al. Cell-Free microRNA-214 From Urine as a Biomarker for Non-Muscle-Invasive Bladder Cancer. Korean J Urol. 2013;54(11):791-796.

22. Wang G, Chan ES, Kwan BC, et al. Expression of microRNAs in the urine of patients with bladder cancer. Clin Genitourin Cancer. 2012;10(2):106-113.

23. Majid S, Dar AA, Saini S, et al. MicroRNA-23b functions as a tumor suppressor by regulating Zeb1 in bladder cancer. PLOS ONE. 2013;8(7):e67686.

24. Wiklund ED, Bramsen JB, Hulf T, et al. Coordinated epigenetic repression of the miR-200 family and miR-205 in invasive bladder cancer. Int J Cancer. 2011;128(6):1327-1334.

25. Wiklund ED, Bramsen JB, Hulf T, et al. Coordinated epigenetic repression of the miR-200 family and miR-205 in invasive bladder cancer. Int J Cancer. 2011;128(6):1327-1334.

26. Shimizu T, Suzuki H, Nojima M, et al. Methylation of a panel of microRNA genes is a novel biomarker for detection of bladder cancer. Eur Urol. 2013;63(6):1091-1100.

27. Pignot G, Cizeron-Clairac G, Vacher S, et al. microRNA expression profile in a large series of bladder tumors: identification of a 3-miRNA signature associated with aggressiveness of muscle-invasive bladder cancer. Int J Cancer. 2013;132(11):2479-2491.
28. Wang S, Xue S, Dai Y, et al. Reduced expression of microRNA-100 confers unfavorable prognosis in patients with bladder cancer. Diagn Pathol. 2012;7:159.

29. Lee H, Jun SY, Lee YS, Lee HJ, Lee WS, Park CS. Expression of miRNAs and ZEB1 and ZEB2 correlates with histopathological grade in papillary urothelial tumors of the urinary bladder. Virchows Arch. 2013.

30. Catto JW, Miah S, Owen HC, et al. Distinct microRNA alterations characterize high- and low-grade bladder cancer. Cancer Res. 2009;69(21):8472-8481.

31. Guancial EA, Bellmunt J, Yeh S, Rosenberg JE, Berman DM. The evolving understanding of microRNA in bladder cancer. Urol Oncol. 2014;32(1):41.e31-e40.

32. Junker K, van Oers JM, Zwarthoff EC, Kania I, Schubert J, Hartmann A. Fibroblast growth factor receptor 3 mutations in bladder tumors correlate with low frequency of chromosome alterations. Neoplasia. 2008;10(1):1-7.

33. Zabolotneva AA, Zhavoronkov AA, Shegay PV, et al. A systematic experimental evaluation of microRNA markers of human bladder cancer. Front Genet. 2013;4:247.

34. Liu Y, Han Y, Zhang H, et al. Synthetic miRNA-mowers targeting miR-183-96-182 cluster or miR-210 inhibit growth and migration and induce apoptosis in bladder cancer cells. PLOS ONE. 2012;7(12):e52280.

35. Yang W, Sun T, Cao J, Liu F, Tian Y, Zhu W. Downregulation of miR-210 expression inhibits proliferation, induces apoptosis and enhances radiosensitivity in hypoxic human hepatoma cells in vitro. Exp Cell Res. 2012;318(8):944-954.

36. Mo M, Peng F, Wang L, Peng L, Lan G, Yu S. Roles of mitochondrial transcription factor A and microRNA-590-3p in the development of bladder cancer. Oncol Lett. 2013;6(2):617-623.

37. Adam L, Zhong M, Choi W, et al. miR-200 expression regulates epithelial-to-mesenchymal transition in bladder cancer cells and reverses resistance to epidermal growth factor receptor therapy. Clin Cancer Res. 2009;15(16):5060-5072.

38. Kozinn SI, Harty NJ, Delong JM, et al. MicroRNA Profile to Predict Gemcitabine Resistance in Bladder Carcinoma Cell Lines. Genes Cancer. 2013;4(1-2):61-69.

39. Drayton RM, Dudziec E, Peter S, et al. Reduced expression of miRNA-27a modulates cisplatin resistance in bladder cancer by targeting the cystine/glutamate exchanger SLC7A11. Clin Cancer Res. 2014;20(7):1990-2000.

40. Jia AY, Castillo-Martin M, Bonal DM, Sánchez-Carbayo M, Silva JM, Cordon-Cardo C. MicroRNA-126 inhibits invasion in bladder cancer via regulation of ADAM9. Br J Cancer. 2014;110(12): 2945-2954.

41. Majid S, Dar AA, Saini S, et al. MicroRNA-1280 inhibits invasion and metastasis by targeting ROCK1 in bladder cancer. PLOS ONE. 2012;7(10): e46743.

42. Itesako T, Seki N, Yoshino H, et al. The microRNA expression signature of bladder cancer by deep sequencing: the functional significance of the miR-195/497 cluster. PLoS One. 2014;9(2):e84311.

43. Uchino K, Takeshita F, Takahashi RU, et al. Therapeutic effects of microRNA-582-5p and $-3 p$ on the inhibition of bladder cancer progression. Mol Ther. 2013;21(3):610-619.

44. Jeffrey SS. Cancer biomarker profiling with microRNAs. Nat Biotechnol. 2008;26(4):400-401.

45. Weber JA, Baxter DH, Zhang S, et al. The microRNA spectrum in 12 body fluids. Clin Chem. 2010;56(11):1733-1741.

46. Wadhwa N, Jatawa SK, Tiwari A. Non-invasive urine based tests for the detection of bladder cancer. J Clin Pathol. 2012;65(11):970-975.

47. Rosenberg E, Baniel J, Spector Y, et al. Predicting progression of bladder urothelial carcinoma using microRNA expression. BJU Int. 2013;112(7):1027-1034.

48. Puerta-Gil P, García-Baquero R, Jia AY, et al. miR-143, miR-222, and miR-452 are useful as tumor stratification and noninvasive diagnostic biomarkers for bladder cancer. Am J Pathol. 2012;180(5):1808-1815. 
49. Hanke M, Hoefig $\mathrm{K}$, Merz $\mathrm{H}$, et al. A robust methodology to study urine microRNA as tumor marker: microRNA-126 and microRNA-182 are related to urinary bladder cancer. Urol Oncol. 2010;28(6):655-661.

50. Tölle A, Jung M, Rabenhorst S, Kilic E, Jung K, Weikert S. Identification of microRNAs in blood and urine as tumour markers for the detection of urinary bladder cancer. Oncol Rep. 2013;30(4):1949-1956.
51. Yamada Y, Enokida H, Kojima S, et al. MiR-96 and miR-183 detection in urine serve as potential tumor markers of urothelial carcinoma: correlation with stage and grade, and comparison with urinary cytology. Cancer Sci. 2011;102(3):522-529.

\section{Publish your work in this journal}

The International Journal of Nanomedicine is an international, peerreviewed journal focusing on the application of nanotechnology in diagnostics, therapeutics, and drug delivery systems throughout the biomedical field. This journal is indexed on PubMed Central, MedLine, CAS, SciSearch ${ }^{\circledR}$, Current Contents ${ }^{\circledR} /$ Clinical Medicine,
Journal Citation Reports/Science Edition, EMBase, Scopus and the Elsevier Bibliographic databases. The manuscript management system is completely online and includes a very quick and fair peer-review system, which is all easy to use. Visit http://www.dovepress.com/ testimonials.php to read real quotes from published authors. 